\title{
Solid charged-core model of ball lightning
}

\author{
D. B. Muldrew \\ 16 Elmsley Crescent, Ottawa, K2H 6V2, Canada
}

Received: 4 May 2009 - Revised: 5 November 2009 - Accepted: 23 November 2009 - Published: 22 January 2010

\begin{abstract}
In this study, ball lightning (BL) is assumed to have a solid, positively-charged core. According to this underlying assumption, the core is surrounded by a thin electron layer with a charge nearly equal in magnitude to that of the core. A vacuum exists between the core and the electron layer containing an intense electromagnetic (EM) field which is reflected and guided by the electron layer. The microwave EM field applies a ponderomotive force (radiation pressure) to the electrons preventing them from falling into the core. The energetic electrons ionize the air next to the electron layer forming a neutral plasma layer. The electricfield distributions and their associated frequencies in the ball are determined by applying boundary conditions to a differential equation given by Stratton (1941). It is then shown that the electron and plasma layers are sufficiently thick and dense to completely trap and guide the EM field. This model of BL is exceptional in that it can explain all or nearly all of the peculiar characteristics of BL. The ES energy associated with the core charge can be extremely large which can explain the observations that occasionally BL contains enormous energy. The mass of the core prevents the BL from rising like a helium-filled balloon - a problem with most plasma and burning-gas models. The positively charged core keeps the negatively charged electron layer from diffusing away, i.e. it holds the ball together; other models do not have a mechanism to do this. The high electrical charges on the core and in the electron layer explains why some people have been electrocuted by BL. Experiments indicate that BL radiates microwaves upon exploding and this is consistent with the model. The fact that this novel model of BL can explain these and other observations is strong evidence that the model should be taken seriously.
\end{abstract}

Keywords. Electromagnetics (Electromagnetic theory; Guided waves) - Meteorology and atmospheric dynamics (Lightning)

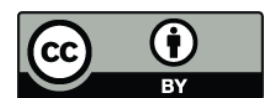

Correspondence to: D. B. Muldrew (dmuldrew@rogers.com)

\section{Introduction}

The characteristics, properties, and observations of ball lightning (BL) are given in books by Singer (1971) and by Barry (1980). A statistical study of data from several data banks on BL is given by Smirnov (1992).

Although high energy BL is rare, there are several examples in the literature of BL with enormous energy content. Goodlet (1937) estimated that a BL that boiled about 17 liters of water had an energy content of about $10^{7} \mathrm{~J}$. Another BL that exploded and destroyed a mud house (Singer, 1971) had an energy content estimated at $4 \times 10^{9} \mathrm{~J}$. A BL (Altschuler et al., 1970) that dug a trench $100 \mathrm{~m}$ long and $1.3 \mathrm{~m}$ deep and tore away $25 \mathrm{~m}$ of stream bank had a conservatively estimated energy greater than $10^{7} \mathrm{~J}$ and a lifetime of $20 \mathrm{~min}$. A large BL (Dmitriev et al., 1981) about $1.5 \mathrm{~m}$ in diameter exploded over the ground producing charred and molten earth over an area $1.5 \mathrm{~m}$ in diameter to a depth of 20 to $25 \mathrm{~cm}$ and evaporated about $175 \mathrm{~kg}$ of water; the energy was estimated to be more than $10^{9} \mathrm{~J}$. Koslov (1978) calculated the BL energy liberated by $\log$ (trees, posts, etc.) destruction to be in the range 10 to $200 \mathrm{MJ}$. According to the virial theorem (Schmidt, 1960), the sum of the kinetic, electric and magnetic energies of a finite plasma cannot exceed $3 p V$ where $V$ is the plasma volume and $p$ is the surface pressure confining it. If atmospheric pressure is solely responsible, then the maximum energy density cannot exceed $3 \times 10^{5} \mathrm{~J} / \mathrm{m}^{3}$.

There are a few models presented in the literature that attempt to explain the high energy. Ashby and Whitehead (1971) suggest that antimatter from space might be responsible for BL; but this model is unable to explain other characteristics such as electrical discharge at termination (Charman, 1979). Altschuler et al. (1970) suggest nuclear reactions; however, it is unlikely that lightning could generate the required isotopes. In other models (Endean, 1976), electromagnetic energy is trapped in an evacuated cavity with an ionized sheath separating it from the atmosphere. This model is appealing but is not compatible with the virial theorem. There are a few models that propose a high-energy loop current either inside or on the surface of BL. Circular currents

Published by Copernicus Publications on behalf of the European Geosciences Union. 


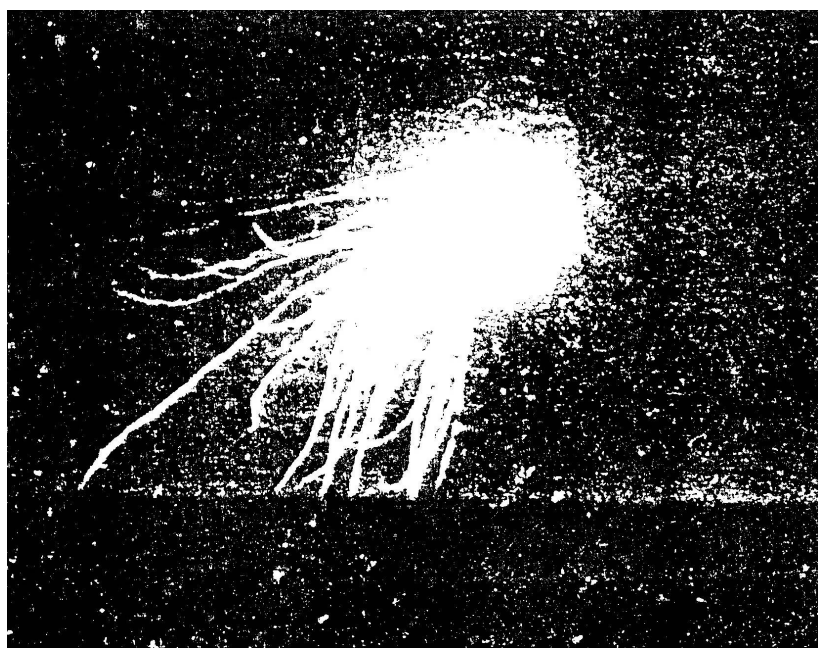

Fig. 1. A photograph of ball lightning spouting sparks. After Kuhn (1951). Singer (1971), reporting Kuhn's observation, states: "The ball, with an estimated diameter of $50 \mathrm{~cm}$... fell directly down and flew apart a few meters above the ground. The photographer reported that he saw the luminous body clearly at the 200-m distance and that there were no other light sources in the field of view". Ground level is about two thirds of the way down from the top of the figure.

have an outward force on the current channel and this outward force must be confined by atmospheric pressure.

Dmitriev et al. (1981) carried out some interesting experiments to determine how the earth beneath the exploding ball lightning could consist of more than a thousand pieces of slag 5 to $6 \mathrm{~cm}$ in diameter and pieces of glass with a fluidlike structure. The original soil consisted of water-saturated sand with quartz grains. They found no changes in the element composition of the soil. Various tests were performed on the original soil to see how the soil was heated. They used irradiative energy, high-frequency electrical discharge, low voltage power sources, high-frequency electromagnetic fields, gasoline, diesel oil, alcohol and thermite. Only the high-frequency electromagnetic field was able to reproduce the observed slag-like material. The frequency used was $13.56 \mathrm{MHz}$; this frequency penetrated the soil to a greater depth than observed. Consequently they added 30\% copper powder to the soil to give the correct penetration depth. They estimated that the frequency required without the copper would be $10^{8}-10^{9} \mathrm{~Hz}$. As will be seen below, this is very convincing evidence that the model presented here is close to the correct model.

Another interesting characteristic of the high-energy ball of Dmitriev et al. is that it spouted sparks. This is a fairly common BL characteristic. These sparks probably correspond to small pieces of the core breaking off due to the intense repulsive force of the charge on the core. These would accelerate radially outward. A photograph (Kuhn, 1951) showing sparks and streamers coming from BL is shown in Fig. 1.
Recent experiments and theories of $\mathrm{BL}$ suggest that $\mathrm{BL}$ is composed of burning nanopartcles (Abrahamson and Denniss, 2000; Abrahamson, 20002; Dikhtyar and Jerby, 2006; Paiva et al., 2007) or of burning vapor or molten metallic spheres (Stephan and Massey, 2008). If the particles or molten spheres have sufficient weight they could counteract the buoyancy force. However there is nothing to keep the BL from expanding or dispersing. BL is known to obtain electrical charge and the charge on the particles would cause them to be repelled from each other and from the ball.

There are several models that consider BL to be a plasmoid (Silberg, 1962) or EM radiation trapped in a plasma sheath (Dawson and Jones, 1969; Endean, 1976). One objection to these models is that these BL should rise like a helium filled balloon. Another objection is that there is nothing containing the plasma. The model presented here has similarities to these models but there is a major difference and that is that BL has a highly charged solid core at its center. This keeps the BL from dispersing into its surroundings and from floating.

The processes involved in BL evolving from a highly charged piece of material to its long-lived quasi steady state is obviously very complicated and the author does not wish to speculate about this. It is thought that an understanding of the quasi steady state should be obtained first and then consideration can be given to the generation mechanism. This is the approach taken here and the generating mechanism is not considered in detail.

\section{Model}

BL is formed by high-energy events such as lightning, volcanoes, tornados, earthquakes and meteors (Singer, 1971). The high energy is capable of generating EM energy and of somehow separating electrons from a small solid body (core) which could be, for example, hail, a stone, or a piece of metal. Electrons separated from the core and those generated by the initiating source, produce an electron layer and highly ionized plasma layer surrounding the positively charged core. These trap the EM energy. If the plasma electron energy is sufficiently high, the electron-ion collision frequency, and hence the absorption of the trapped EM energy by the plasma, is low enough (discussed below) that the ball lifetime could be seconds or more.

The EM energy is guided around the inside of the BL by the electron and plasma layers and hence applies a ponderomotive force (i.e. radiation pressure) to the electrons that prevents them from falling into the positively charged core. A sketch of the model is shown in Fig. 2. A hypothetical BL is considered with a radius of about $10 \mathrm{~cm}$; the core has a radius $a$ of about 0.1 to $2 \mathrm{~cm}$ and a charge $Q$ of about $10^{-5}$ to $10^{-3} \mathrm{C}$. The EM field is of the "whispering-gallery" type (Budden, 1961a). The electron layer has a charge very nearly equal in magnitude to that of the core and a thickness less 
than about $1 \mathrm{~cm}$. The trapped EM energy energizes the electrons to the $\mathrm{keV}$ or tens of $\mathrm{keV}$ level. These completely strip orbital electrons from atmospheric atoms that they come in contact with to produce a neutral plasma layer. For simplicity, the plasma layer is assumed to consist only of energetic electrons and ions of nitrogen $\mathrm{N}^{+7}$. As the plasma layer varies from fully ionized to non-ionized is obviously of extreme complexity and even an approximate description will not be attempted here. It should be noted that Dmitriev (1969) found large concentrations of ozone and nitrogen dioxide in air samples capture from the "bluish mist" trail of a BL.

In a mathematical description of this model given by Muldrew (1990), referred to as Paper 1 below, both a first-order theory and second-order theory are presented. In the first order theory it is assumed that the electron density is zero for $a<r<b$ where $r$ is distance measured from the center of the ball (see Fig. 2). For $r>b$ it is assumed that the electron density is constant and is determined by equating the plasma pressure where the EM field is small, to atmospheric pressure $p_{0}$. In the second-order theory, three differential equations are obtained for the self-consistent electron density, ion density, and EM electric field distributions as a function of $r$. These are solved numerically for a particular case. In Eq. (2.3) of Paper 1 the plasma pressure is given by the sum of the electron and ion pressures. Equating this to atmospheric pressure $p_{0}$ gives

$p_{0}=N K T_{e}+N_{i} K T_{i}=K N\left(T_{e}+T_{i} / Z\right)$

where $N$ and $T_{e}$ are the electron density and temperature, $N_{i}$ and $T_{i}$ are the ion density and temperature, $K$ is Boltzmann's constant and $N_{i}=N / Z$. Equation (1) is valid if the mean free path is small compared to the distance over which the macroscopic properties of the plasma vary or if there is a magnetic field for which the cyclotron radius is small compared to this distance (Spitzer, 1962). The electron mean free path is extremely large (Paper 1) and a magnetic field, which may be present due to currents, is not considered. If the plasma pressure is only due to the ions, then

$p_{0}=N_{i} K T_{i}=N K T_{i} / Z$

In reality, the electron density $N$ would be between that determined from Eq. (1) and that determined from Eq. (2). The effect of this on the BL model presented in Paper 1 is that the electron density in the electron and plasma layers is no longer limited by the electron temperature. The ball energy and the electric field distribution inside the electron layer for the example shown in Fig. 2 of Paper 1 is not greatly affected but, due to the higher electron density, much higher EM frequencies can be trapped by the ball. This means that smaller balls can be obtained than predicted by the model of Paper 1 . The minimum size, of about $1 \mathrm{~cm}$, is then determined by the strength and size of the core and agrees fairly well with observation. Calculations based on this modification indicate

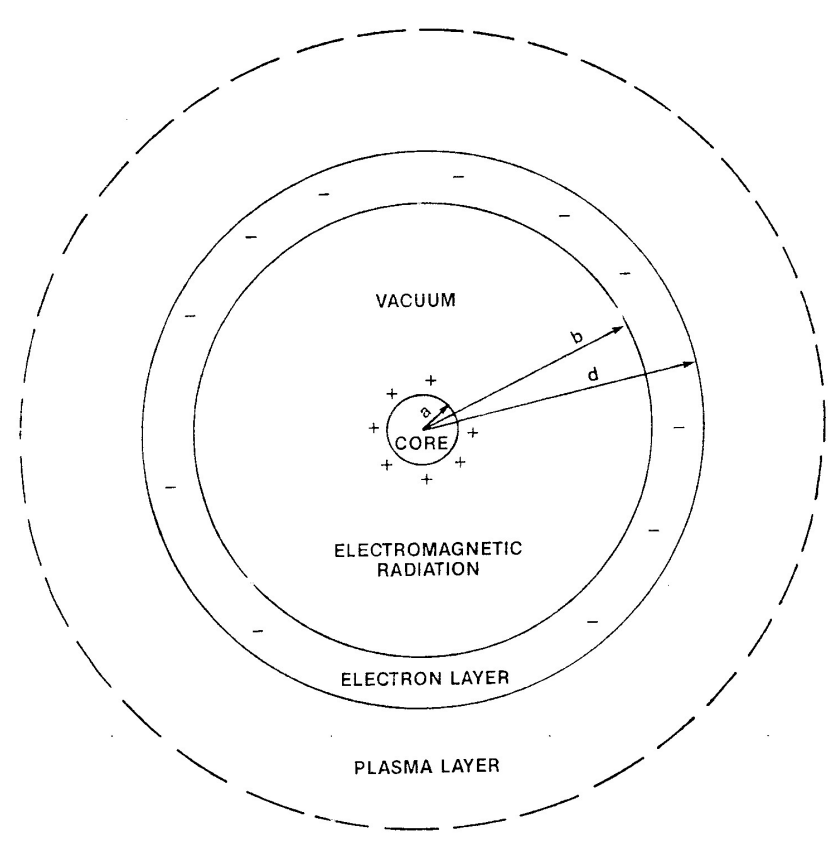

Fig. 2. A sketch of the ball lightning model. After Fig. 1 of Muldrew (1990).

that small-size BL can be described by the model (see below).

An error in Fig. 2 of Paper 1 should be pointed out here. The electron layer in Fig. 2 of Paper 1 should be about $0.34 \mathrm{~cm}$ thick, not about $0.12 \mathrm{~cm}$ thick, as shown in the figure. The text and calculations are correct.

In the second-order theory of Paper 1, the first term on the left-hand side of Eq. (3.1) is the electron pressure-gradient term and, as discussed above, should be modified. The results presented in Fig. 3 of Paper 1 would then be different.

\section{Evidence for a solid core}

The feasibility of the above model can be demonstrated by presenting the observational evidence that BL has a solid core.

In the solid charged-core model of BL presented here, the atmosphere confines a small amount of energy but for energetic balls, nearly all of the energy is confined by the strength (molecular bonding energy) of the core. Consider a core with a positive electric charge distributed over its surface, as would be the case for a metallic or conductive core. The positive charge produces an outward repulsive electrostatic force over the surface tending to tear the core apart. The virial theorem applies to the core. The electrostatic energy, which can be confined by the core, is $3 p V$ where $V$ is the volume of the core and $p$ is the outward electrostatic pressure at the surface of the core required to break it apart. The total energy confined by the core can be orders of magnitude greater than the energy that can be confined by atmospheric pressure alone. 
Strong steel has a tensile strength of about $3 \times 10^{9} \mathrm{~N} / \mathrm{m}^{2}$. Tensile strength is determined by stretching a wire to the breaking point and then dividing by the cross sectional area. In this process, the cross sectional area decreases as the length of the wire increases. For a solid such as a sphere, with an outward radial force applied to the surface, this cannot happen since the dimensions normal to the applied force must also increase if the radial dimension is increased. It thus seems reasonable that the conventional tensile strength is not appropriate in this case. The bonding force between molecules of the material may determine the limiting force. This can be estimated from the amount of heat energy required to raise the material to its boiling point and evaporate it. This would of course over-estimate the breaking strength because of flaws in the material. For iron the bonding energy is about $7 \times 10^{10} \mathrm{~J} / \mathrm{m}^{3}$ and applying the virial theorem gives a bonding strength of about $2 \times 10^{10} \mathrm{~N} / \mathrm{m}^{2}$. If a strength of $10^{10} \mathrm{~N} / \mathrm{m}^{2}$, say, is chosen for the strength of a spherical steel core, then a BL energy of $10^{9} \mathrm{~J}$ could be contained by a sphere about $17 \mathrm{~cm}$ in radius. It is quite possible that the energy of the most energetic BL was over estimated by, say, a factor of 10 . For an energy of $10^{8} \mathrm{~J}$, the spherical steel core would be about $7 \mathrm{~cm}$ in radius and would weigh about $11 \mathrm{~kg}$. The buoyancy force on a $1 \mathrm{~m}^{3} \mathrm{BL}$ is only about $1 \mathrm{~kg}$, and hence energetic BL would be considerably heavier than air. This is borne out by the observations: In the Goodlet (1937) example the BL fell from a house roof into a container of water. Obviously, in the Altschuler et al. (1970) example the BL must have had considerable weight to dig a trench $1.3 \mathrm{~m}$ deep. In the Dmitriev et al. (1981) example the BL appeared over a cinema and then descended to the earth's surface through tree branches. Hence, even though the core is large it is not unlikely that the highest estimated levels of BL energy can be explained by the solid-core model.

BL is usually generated by lightning. Lightning striking an object on the ground could break off a piece of the object and electrically charge it due to the intense electric currents and/or electric fields associated with the lightning channel. This could form the core of BL. During thunderstorms BL is sometimes observed falling from clouds or observed from aircraft. In this case the core could be a piece of a meteor. An ionized meteor trail would form a good path for lightning and hence it is not that unlikely that a meteor would be hit by lightning during a storm. BL observed inside or just outside an aircraft could be caused by lightning striking or occurring near the aircraft and producing a large current or electric field in some material inside or outside the aircraft. A piece of this material could become the core. Clear air BL is also occasionally seen; in fact, the author has observed this type of BL and thinks the two balls observed resulted from meteor fragments. A large meteor was observed about the same time but the exact time relative to the ball lightning observations is uncertain due to fading memory. Material ejected from volcanoes, picked up from the ground by tornados and hurricanes, or broken off of meteors would be subjected to severe frictional forces resulting in electrostatic charging. Piezoelectric effects near the fault of an earthquake produce intense electric fields that could highly charge a piece of material. This material would form the core of BL. The core would normally have an irregular shape and if its size were an appreciable fraction of the ball diameter, it could produce a nonspherical ball. Nonspherical balls are observed in perhaps 15\% of the reported observations (Singer, 1971).

Jones (1910), Potts (1910) and others give direct evidence of BL having a solid core. One of Jones' students shorted an ordinary (for that period) $110 \mathrm{~V}$ circuit. Jones writes:

"I happened at the time to be a few meters from him and to be looking toward the terminals. At the instant of the short circuit I saw an incandescent ball which appeared to roll rather slowly from the terminals across the laboratory table and then disappeared. As I remembered it, I should say that the ball may have appeared to be about three centimeters in diameter. I think no one else in the room saw anything more than a flash of light - much as if a fuse had blown. On the table where the ball had rolled we found a line of scorched spots, as if the ball had bounced along the table and had scorched the wood wherever it touched. As I remember them, these scorched spots were rather close together, perhaps not much more than one or two centimeters apart. In the top of the table was a crack perhaps a millimeter or two wide, and at this crack the scorched line ended. In a drawer immediately under the crack we found a tiny copper ball, perhaps a millimeter in diameter. Apparently the ball that rolled along the table was incandescent copper vapor, although my memory of it is rather like a yellow-white than a greenish light."

The observations of Jones are discussed in more detail below. Potts writes of his observation during a heavy electrical storm:

"I observed what appeared to be a ball of fire between two and three feet in diameter rolling along the street. It was also accompanied by several others of smaller size. This appearance occurred just after a very heavy electrical discharge to a telephone pole some few squares away. The discharge along the telephone wire heated the wire to red heat. The wire broke on account of this heating and a section of some considerable length was hurled along the street with a whirling motion. The rapidity of the rolling motion gave the appearance of a ball, as it also gave a forward motion to the ball of fire. Subsequent investigation revealed the two ends of the wire dangling from adjacent poles with a considerable length of wire missing."

R. Golka (private communications, 1993; 1994) apparently generated small BL from high-current, low-voltage arcs under water. These BL danced on the water surface for a short time and when they dissipated, a roughly spherical piece of metal remained with a diameter of about $1 \mathrm{~mm}$. Although Golka may not agree, it is thought that this piece of metal was the BL core. Resin was found in a fulgurite resulting from ball lightning that originated in a lightning flash to 
a tree (Meunier, 1886). During a lightning storm, a tar-like substance was reported on the ground from a burning ball that exploded after half a minute (Webber, 1907). Witnesses observed a large ball of fire formed by lightning "vaporizing" a $65-\mathrm{cm}$ length of a 2-mm copper antenna wire (Turpain, 1911). Large objects have been seen to fall from energetic ball lightning (Corliss, 1982). Sparks and streamers emanating from BL (Fig. 1) also indicate that BL has a solid core.

All of these observations are evidence that BL contains a solid core. In most cases a core is not observed but this does not mean that one didn't exist. It could be too small to notice, it could blow apart during explosion (Fig. 1), it could vaporize, or it could be dispersed upon melting.

\section{Theory}

The first-order theory will be presented here with the appropriate modification described above and with more detail and clarification. The second order-theory will not be presented here. It is, of course, interesting to know the detailed electron density, ion density and EM field distributions in BL but it is not essential for an understanding of the model. In the second order theory it is only necessary to assume a positive core surrounded by an electron layer of equal but opposite charge. In the first order theory the electron density $N$ is assumed to be zero inside a radius $b$ and for all $r>b, N$ is assumed constant. This is justified based on the second order theory. From $r=b$ to $r=d$, there is an electron layer that contains a total charge $-Q$ assumed equal in magnitude to that of the core. For $r>d$ there is a neutral plasma layer of fully ionized nitrogen ions $\mathrm{N}^{+7}$. The electrons in the electron layer have energies of order of tens of keV. It takes about $10 \mathrm{eV}$ to ionize an air molecule and hence the air near the outer boundary of the electron layer would be ionized by the energetic electrons.

Equations (8) on page 556 of Stratton (1941) for the boundary conditions of the EM oscillations of a sphere, are now used to obtain the natural angular frequency $\omega$ of the trapped EM energy. For $r<b$ the wave number $k_{1}$ is that of free space $\omega / c$ and for $r>b$ the wave number is (Budden, 1961b)

$k_{2}=\frac{\omega}{c}\left[1-\frac{e^{2} N}{\varepsilon_{0} m \omega^{2}}\right]^{\frac{1}{2}}$

where $c, e, m$ and $\varepsilon_{0}$ have their usual meaning. It is assumed Eq. (3) is valid even though due to the high electron temperature (see below), the electrons are slightly relativistic.

The tangential component of the EM electric field is continuous at $r=b$. Hence, assuming the permeability of the

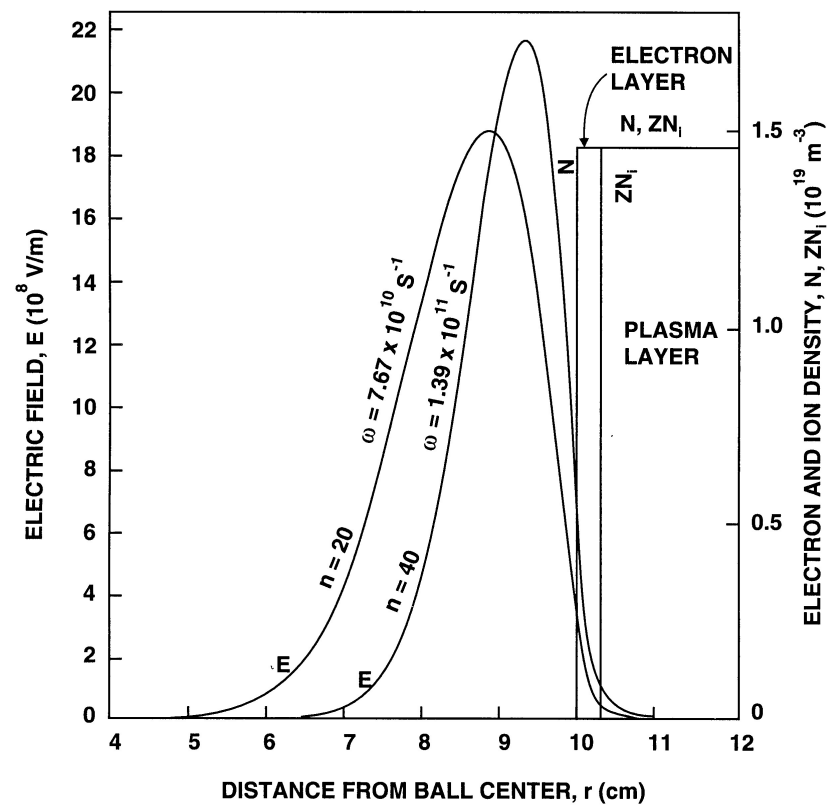

Fig. 3. Electric-field distributions for the fundamental $n=20$ and $n=40$ modes assuming electron and plasma layers of constant electron density. After Fig. 2 of Muldrew (1990) with corrected electron layer thickness.

plasma is that of free space, from Eq. (10), page 556, of Stratton

$\frac{\left[k_{1} b j_{n}\left(k_{1} b\right)\right]^{\prime}}{j_{n}\left(k_{1} b\right)}=\frac{\left[k_{2} b h_{n}^{(1)}\left(k_{2} b\right)\right]^{\prime}}{h_{n}^{(1)}\left(k_{2} b\right)}$

where $j_{n}$ and $h_{n}^{(1)}$ are spherical Bessel and Hankel functions. For $r<b$, the electric field is proportional to $j_{n}\left(k_{1} r\right)$ and for $r>b$, the electric field is proportional to $h_{n}^{(1)}\left(k_{2} r\right)$. The primes in Eq. (4) denote differentiation with respect to $k_{1} b$ and $k_{2} b$. Numerical calculations involving spherical Bessel and Hankel functions are carried out using the formulae given on page 405 of Stratton. The electric field distribution for $n=20$ and for $n=40$ are shown in Fig. 3. The plasma parameters used in the calculation are discussed below. The wave frequencies are determined by the boundary conditions.

The assumed constant value of $N$ is found by equating the plasma pressure to atmospheric pressure $p_{0}$. The minimum value of $N$ is given by Eq. (1) and the maximum value of $N$ by Eq. (2). Calculations have been carried out for various values between these two limits.

The electrons oscillate in an intense EM field and occasionally collide with ions converting EM energy into thermal energy. The thermal energy is determined by equilibrium between heat input from the EM field due to collisions and heat loss due to radiation and conduction to the surrounding air. The electron temperature is consistent with the intense EM field. It is shown below that the electron temperature is about $10^{9} \mathrm{~K}\left(10^{2} \mathrm{keV}\right)$ and hence there is little interaction 
between electrons and ions; consequently the ions have their own temperature. Preliminary calculations (not given here) using Banks and Kockarts (Part B, 1973) indicate that if the ion temperature is assumed constant, it cannot exceed a few thousand degrees since otherwise energy loss due to thermal conduction would be large; $T_{i}$ is taken to be $5000 \mathrm{~K}$.

The order $n$ of the spherical functions determines the shape of the radial electric field. The greater $n$ is, the smaller the field at $r=a$ is. If the core is conductive, a strong field at the core surface could produce sufficient ohmic heating to melt the core. A small field at the core is achieved by choosing $n$ greater than about 10 . For the calculations presented here, $n=20$ and $n=40$. It also is assumed that the EM field depends only on $r$. The field is chosen to be independent of the azimuth angle $\phi$ by choosing the index $m=0$ (see Stratton, page 555). As a function of the latitudinal angle $\theta$, however, the electric field has $n$ nulls. Such a field could be set up, for example, by a single VLF transmitting source in the earth-ionosphere waveguide (Budden, 1961a). But suppose there were two separated VLF transmitting sources. In a linear system, both would have their own system of modes with differently located nulls. The two fields would of course interfere producing a complicated pattern of electric field minima and maxima. However, in general, the minima would not be nulls. This corresponds in some way to the present problem where there is no particular source or perhaps there is an extremely large number of small, evenly distributed sources. The average electric field due to all the different sources and all the different modes would tend to average out leaving no nulls but possibly leaving electric field minima. It therefore seems justifiable, to a first approximation, to take the electric field as a function of $r$ only. The EM field in the ball corresponds to what is commonly referred to as the "whisperinggallery' mode of propagation (Budden, 1961a).

Given $N$ and $b$, Eq. (4) is solved numerically for $\omega$ using Eq. (3). Since $\omega$ is multivalued, the smallest value is chosen. This value results in a solution for which there is only a single maximum in $|E|$ between $r=0$ and $r=\infty$ where $E$ is the electric field strength.

The constant of proportionality between $E$ and $j_{n}\left(k_{1} r\right)$ for $r<b$ is determined from the charge on the core $Q$. One method of finding it is to use the virial theorem. The desired form of the virial theorem can be obtained from Eq. (13) of Schmidt (1960). For a steady state, the left-hand side of this equation is zero. Consider a sphere with a constant scalar gas pressure $p$ outside a radius $b$. If the gas has no macroscopic flow $(T=0)$ and a vacuum exists for $r<b(U=0)$, then the enclosed magnetic and electric electromagnetic en$\operatorname{ergy} E^{M}+E^{E}$ is

$S_{E M}=E^{M}+E^{E}=b p \int d S=4 \pi b^{3} p=3 V p$

where $S_{E M}$ is the total enclosed EM energy of a ball of volume $V$ having a pressure $p$ at the surface $S$. The enclosed energy does not include the electrostatic (ES) energy of the charge on the core since this energy is confined by the strength of the core itself. Assuming the electron layer is very thin, Eq. (5) gives

$$
\begin{aligned}
S_{E M} & =2 \int_{a}^{\infty} \frac{1}{2} \varepsilon_{0} E^{2} 4 \pi r^{2} d r \\
& =3\left(\frac{4}{3} \pi b^{3}\right)\left[p_{0}+\frac{Q^{2}}{8 \pi \varepsilon_{0} b^{2}\left(4 \pi b^{2}\right)}\right]
\end{aligned}
$$

where the second term in square brackets on the right is the ES pressure of the electron layer and $p_{0}$ is atmospheric pressure. The electric field energy $E^{E}$ is given by the integral; the energy density of the EM electric field is $(1 / 2) \varepsilon_{0} E^{2}$ and $4 \pi r^{2} d r$ is a volume element. The 2 in front of the integral results since $E^{E}=E^{M}$. Let

$E=\frac{E_{0}}{j_{n}\left(k_{1} b\right)} j_{n}\left(k_{1} r\right)$

Then $E_{0}$, the value of $\mathrm{E}$ at $r=b$, is determined from Eq. (6) numerically.

Equation (6) is of interest historically since much has been written in the literature regarding $\mathrm{BL}$ and the virial theorem. However, there is a better method to determine $E_{0}$. The ponderomotive force on a single electron is $-e^{2} /\left(2 m \omega^{2}\right)\left(d E^{2} / d r\right)$ (Fejer, 1979). The ponderomotive force on the electron and plasma layers is balanced by the force of electrostatic attraction of the electron layer to the core plus the force of atmospheric pressure. Hence for a thin electron layer near $r=b$,

$-\int_{b}^{\infty} \frac{e^{2}}{2 m \omega^{2}} \frac{d E^{2}}{d r}\left(4 \pi r^{2} N d r\right)=4 \pi b^{2} p_{0}+\frac{Q^{2}}{8 \pi \varepsilon_{0} b^{2}}$

where the quantity in brackets is the number of electrons in $d r$. If $E$ falls rapidly to zero for $r>b, 4 \pi r^{2}$ can be set equal to $4 \pi b^{2}$ and from Eq. (7)

$-\int_{b}^{\infty} \frac{d E^{2}}{d r} d r=-\int_{E_{0}^{2}}^{0} d E^{2}=E_{0}^{2}$

From Eqs. (8) and (9)

$$
\frac{1}{2} \varepsilon_{0} E_{0}^{2}=\frac{\omega^{2}}{\omega_{p}^{2}}\left[p_{0}+\frac{Q^{2}}{2 \varepsilon_{0}\left(4 \pi b^{2}\right)^{2}}\right]
$$

where the angular plasma frequency $\omega_{p}=\left[e^{2} N /\left(\varepsilon_{0} m\right)\right]^{1 / 2}$.

From Eq. (10), $E_{0}$ is determined for $Q=10^{-3} \mathrm{C}, N=$ $1.45 \times 10^{19} \mathrm{~m}^{-3}$ (this density has been chosen equal to the value calculated in Paper 1 in order that the results be unchanged), $b=0.1 \mathrm{~m}$ and for $n=20$ and $n=40$, and $E(r)$ is found from the Bessel and Hankel functions using Eq. (7). The results are shown in Fig. 3. A core charge of $10^{-3} \mathrm{C}$ is 
chosen to show that the model is capable of explaining energetic BL. Lower energy BL is much more common and would have a core charge about $3 \times 10^{-6}$ to $10^{-4} \mathrm{C}$.

Suppose, hypothetically, that the core consists of a spherical steel ball of radius $a$ and with strength sufficient to carry a charge density $\rho=0.3 \mathrm{C} / \mathrm{m}^{2}$. Then, $4 \pi a^{2}=Q / \rho$ or $a=0.016 \mathrm{~m}$ and the core mass is about $0.15 \mathrm{~kg}$. The ES energy $S_{E S}$ is

$S_{E S}=\frac{Q^{2}}{8 \pi \varepsilon_{0}}\left(\frac{1}{a}-\frac{1}{b}\right)$

or $2.36 \times 10^{5} \mathrm{~J}$ and an electron in the electron layer has an average potential energy of about $240 \mathrm{MeV}$. The EM energy can be obtained from Eq. (6).

$S_{E M}=\frac{Q^{2}}{8 \pi \varepsilon_{0} b}+4 \pi b^{3} p_{0}$

or $4.6 \times 10^{4} \mathrm{~J}$. Neglecting the thermal energy of the electrons and the vacuum energy, the total energy is from Eqs. (11) and (12)

$S_{T}=\frac{Q^{2}}{8 \pi \varepsilon_{0} a}+4 \pi b^{3} p_{0}$

or about $2.8 \times 10^{5} \mathrm{~J}$.

The outward pressure at the surface of the core is

$p_{s}=\frac{Q^{2}}{8 \pi \varepsilon_{0} a^{2}\left(4 \pi a^{2}\right)}$

or $5 \times 10^{9} \mathrm{~N} / \mathrm{m}^{2}$. This is near the upper limit for the strength of steel (see above).

The electron-ion collision frequency and bremsstralung in the plasma layer are dependent on the electron temperature. Equating the electron energy corresponding to a temperature $T_{e}$ to the electron energy in the EM field gives

$\frac{3}{2} K T_{e}=\frac{1}{2} m\left(\frac{e E}{m \omega}\right)^{2}$

As an example, for an $E$ of about $10^{8} \mathrm{~V} / \mathrm{m}$ and $\omega$ about $10^{11} \mathrm{~s}^{-1}$, a $T_{e}$ of about $10^{9} \mathrm{~K}$ (about $10^{2} \mathrm{keV}$ ) is obtained. The electron energy is lost by bremsstrahlung, ionization, dissociation and conduction in the plasma layer. Also, the most energetic electrons will escape from the ball. This will leave a small net positive charge on the ball which will constrain the less energetic electrons to orbit the core. In any case the electron energy is so large that energy losses due to electron-ion collisions can probably be neglected in determining ball lifetime for medium and large-size BL, and allows lifetimes of about $1 \mathrm{~s}$ for BL of diameter down to about $1 \mathrm{~cm}$. The energy loss means that we do not have a true steady state.

\section{The observations of Jones}

The first order theory of Paper 1 was applied to the observation of Jones (1910). By trial and error a particular model ball was found which agrees quite well with the observation of Jones. Using the same symbols as in Paper 1, the values are: $Q=3 \times 10^{-6} \mathrm{C}, b=1.7 \mathrm{~cm}, n=4, N=10^{20} \mathrm{~m}^{-3}$, where $Q$ is the core charge, $b$ is the ball radius, $n$ is the order of the spherical functions describing the trapped electromagnetic field, and $N$ is the electron density of the electron and plasma layers. The tensile strength for copper is about $4 \times 10^{8} \mathrm{~N} / \mathrm{m}^{2}$. As mentioned above, the strength on the surface of a sphere of copper would be greater than this. The molecular bonding strength (see above) yields a surface strength of about $1.5 \times 10^{10} \mathrm{~N} / \mathrm{m}^{2}$. A strength midway between these of $3 \times 10^{9} \mathrm{~N} / \mathrm{m}^{2}$ is chosen for the calculations. From this value and $Q$ a radius for the core is calculated to be $1.0 \mathrm{~mm}$ and the associated electrostatic energy $49 \mathrm{~J}$. The angular frequency of the trapped EM radiation is $1.4 \times 10^{11} \mathrm{~s}^{-1}$. If the core is spherical and located at the center of the ball then, for the above parameters, the EM field at the surface of the sphere is sufficiently small so that it will take several tens of seconds for the core to melt by ohmic heating.

In Paper 1 it was suggested that for a freely-moving ball the electrons in the electron layer would follow the motion of the core since they are to some extent in orbit about the core. In Jones' example the ball motion is restricted by the table. Hence there must be forces acting on the core to keep it centered. The ponderomotive force (radiation pressure) of the trapped EM field acting on the free electrons of the conductor is certainly great enough to do this (Stratton, 1941). One problem is that if the ponderomotive force on the core were sufficient to, say, balance the gravitational force, the ohmic heating might be so great that the ball would melt. There is another force which is probably greater than the ponderomotive force and which would prevent the ball from melting. The EM field increases very rapidly with distance from the center of the ball if $n$ is not too close to 1 . The surface of the core furthest from the center of the ball would encounter the largest EM field. If a portion of the surface moves into a high EM field it could be vaporized. The momentum of the escaping vapor would apply a force on the core and push it back toward the center. This would keep the core from melting due to ohmic heating and would result in producing an approximately spherical core as was observed by Jones and by Golka (see above).

\section{Ball lightning motion}

One of the remarkable observations of BL is that it often appears to float about $1 \mathrm{~m}$ or less above the ground or floor. In fair weather the vertical electric field a few meters above the Earth's surface is about $100 \mathrm{~V} / \mathrm{m}$ and downward so that a positively charged ball would experience a small downward 
force due to this field. However, during storm conditions, when BL is usually observed, the electric field is reversed and about $10000 \mathrm{~V} / \mathrm{m}$. There is thus an upward force on a positively charged BL. Within about $1 \mathrm{~m}$ of a surface the total positive plus negative ion concentration in the atmosphere is reduced by about a factor of two due to collision of the ions with the surface. This decreases the conductivity and increase the vertical electric field by a factor of about two. This is called the electrode effect (Hopple, 1967). A BL falling toward the Earth's surface during storm conditions will thus experience an increasing upward force in about the last meter. The buoyancy, gravitational and electrostatic forces may then balance within about $1 \mathrm{~m}$ of the surface. Consider a $10-\mathrm{cm}$ radius ball with a core mass of $10 \mathrm{~g}$. The upward buoyancy force is about $4 \mathrm{~g}$ weight. The downward $6 \mathrm{~g}$ net weight is balanced by an upward electric field of, say, $15000 \mathrm{~V} / \mathrm{m}$ for a net positive charge on the ball of $4 \times 10^{-6} \mathrm{C}$, a reasonable net charge.

The distortion of the Earth's electric field by the walls surrounding windows and doors of a building can also explain the tendency for BL to enter and leave buildings through open windows and doors. A particularly strange but common observation is that $\mathrm{BL}$ enters a building through the chimney. Smoke is more highly ionized than normal air. Hence, in a smoke trail from a chimney the conductivity is higher and the electric field is lower than in the background air. The trail could thus act as a duct and guide the ball into the chimney. Another peculiar observation is that BL has a tendency to follow aircraft. An aircraft would likely attain a high electrostatic charge in its motion through the air, especially during electrical storms. Due to the shape of the aircraft and to interference with the Earth's field, electric field minima would exist in the vicinity of the aircraft. These minima could trap a BL causing it to follow the aircraft.

\section{Discussion}

There are several examples of BL having electrical properties similar to lightning. In fact, people have been injured or killed by electrical discharges from BL. The charged core model of BL can explain this; nearly all other proposed models cannot.

The strong microwave radiation trapped in BL and escaping upon explosion can explain the experimental results of Dmitriev et al. (1981). They estimated the microwave frequency that heated the soil to be about $10^{8}-10^{9} \mathrm{~Hz}$. The diameter of the observed BL the studied was about $1.5 \mathrm{~m}$ or 7.5 times the diameter of the BL of Fig. 2. The frequencies corresponding to the $n=20$ and $n=40$ examples in Fig. 2 are about $1.2 \times 10^{10}$ and $2.2 \times 10^{10} \mathrm{~Hz}$. Scaling these frequencies down for the $1.5 \mathrm{~m} \mathrm{BL}$ gives $1.6 \times 10^{9}$ and $2.9 \times 10^{9} \mathrm{~Hz}$. These frequencies are higher than the range given by Dmitriev et al. but choosing a smaller value of $n$ would bring the value into their range. This gives very strong support for the solid-charged core model of BL.

The electron temperature $T_{e}$ must be sufficiently great that the electron energy loss due to EM damping in the BL lifetime is considerably less than the total ball energy. From Eq. (3.42) of Budden (1961b), the power loss per unit volume due to collisions $W_{c}$ in a time $t \gg 1 / \omega$ and for $\omega \gg v$, where $v$ is the momentum collision frequency, is

$W_{c}=\frac{N e^{2} v E^{2}}{m \omega^{2}}$

where $E$ is the root-mean-square value of the field and not the peak value used by Budden. For electron-ion collisions (Banks and Kockarts, Part A, 1973), using mks units

$v=3.8 \times 10^{-5} N_{i} Z^{2} T_{e}^{-3 / 2}$

Bank and Kockarts give the energy collision frequency which is twice the momentum collision frequency; their value must thus be divided by 2. An error was made in Eq. (4.1) of Paper 1. For reasonable values of $N_{i}$ and $T_{e}$, a value of $\ln (\Lambda)$ (Table 5.1, Spitzer, 1962) of 21 is more appropriate than the value 15 used by Banks and Kockarts. The total EM power loss $P_{c}$ is from Eqs. (16) and (17)

$P_{c}=\frac{3.8 \times 10^{-5} Z^{2} e^{2}}{m \omega^{2} T_{e}^{3 / 2}} \int_{a}^{\infty} N N_{i} E^{2} 4 \pi r^{2} d r$

For $n=20$ and $n=40$, in the example of Fig. 3, numerical integration yields $P_{c}=1.8 \times 10^{3} \mathrm{~W}$ and $P_{c}=2.5 \times 10^{3} \mathrm{~W}$, respectively for $T_{e}=5 \times 10^{8}$. From an electron-ion collision point of view the "half-life" of the ball, $S_{T} / P_{c}$, is from Eqs. (12) and (18) then about $150 \mathrm{~s}$ for $n=20$ and $110 \mathrm{~s}$ for $n=40$. Lower energy balls would have shorter electron-ioncollision lifetimes; but there are also other factors which determine lifetime. The BL model presented here explains one of the most puzzling characteristic of BL - its long lifetime.

Ignoring the relatively small atmospheric pressure term in Eq. (13), the total energy $S_{T}$ is independent of $b$. As the EM energy is converted to heat, the only way to decrease $S_{T}$ is to decrease $Q$, since $a$ is unlikely to change significantly. Starting with a ball in equilibrium, after EM energy is lost, the ES and atmospheric pressures are greater than can be supported by the trapped EM energy. Since observationally, the ball doesn't collapse immediately, it appears that either small charged particles break off and are repelled from the core ("sparks" are often observed ejected from a ball) or electrons from the electron layer penetrate the EM barrier and partly neutralize the core. Electron penetration could result from small, local instabilities and/or from interference minima in the EM field. The EM field of BL probably consists of many interfering modes covering a fairly large frequency range, not one mode as considered here.

The size of BL has been observed to remain the same throughout its lifetime. This is a surprising observation since intuitively one might expect size to change as the ball energy 
decreases. However, from Eq. (13) it can be seen that the size of the ball $b$ does not change significantly as the total energy $S_{T}$ changes since the second term on the right is small relative to the first term.

$\mathrm{BL}$ often terminates in an explosion. This might be due to an uncontrolled instability and/or melting of the core material by a transfer of ES energy to heat. The ES energy of an electron penetrating the EM field would be dissipated by radiation (analogous to synchrotron radiation), mainly in the visible and ultraviolet, as the relativistic electron spirals into the core, and by subatomic particle generation in collision with the core.

Radiation (bremsstrahlung) is generated in the plasma layer. The radiated power per unit volume is obtained from Eq. (5-60) of Spitzer (1962). In mks units, with $N=Z N_{i}$

$$
W_{R}=1.4 \times 10^{-40} Z N^{2} T_{e}^{1 / 2}
$$

The thickness of the plasma layer $\Delta s$ is not known but presumably is much less than $b$. Then, for $T_{e}=5 \times 10^{8} \mathrm{~K}$, $b=0.1 \mathrm{~m}, Z=7, N=1.45 \times 10^{19}$ the power loss $P_{R}=$ $W_{R} 4 \pi b^{2} \Delta s=6 \times 10^{4} \Delta s \mathrm{~W}$. For $\Delta s<0.01 \mathrm{~m}, P_{R}<600 \mathrm{~W}$ which is considerably less than the collisional loss of Eq. (18) for the chosen $T_{e}$. Of this, a few milliwatts are radiated in the visible, about $1 \mathrm{~W}$ in the ultraviolet and the rest in $\mathrm{X}$ rays (Spitzer, 1962). An interesting observation noted by Dmitriev (1969) in a footnote is that gamma radiation may have been detected from the BL by a gamma radiation scintillation radiometer located close to the BL. There are, of course, other sources of visible light: mainly recombination but also the 'synchrotron' radiation mentioned above.

The BL model presented here has been kept simple (i.e. a single EM mode) to allow a mathematical description. A more elaborate model is not justified on the basis of the observational data. In reality, BL would be more complicated. For example, the EM field could consist of many interfering modes covering a large frequency range. Interference minima could allow leakage of electrons through the EM barrier. Also, as mentioned above, the plasma layer would be of extreme complexity. Air molecules diffuse inward becoming dissociated and ionized. The inward flux of neutrals and consequent energy loss to the ball is reduced by an equal outward flux of ions (cf. a candle flame).

In spite of the complexity of this model, it is still thought that it is fundamentally correct but still incomplete. It explains the large amount of energy associated with BL without violating the virial theorem or resorting to unusual forms of matter. The author knows of no other model that can do this. It also explains other unusual characteristics such as lifetime, electrical properties, and motion. A small net charge on a ball interacts with the earth's natural electric field (which can be quite strong during thunderstorms) or other local electric fields (e.g. near aircraft) to produce erratic motion. Because of the weight of the core, there is no buoyancy problem as there is with gas or plasma models.

\section{Conclusions}

The following conclusions and predictions are based on this paper and Paper 1. The predictions could be tested if a technique for artificially producing $\mathrm{BL}$ is found.

1. There is a minimum size for BL. The theoretical and observed values of about $1 \mathrm{~cm}$ are reasonably close. Using Eqs. (12) and (18) the half-life of a ball based on absorption of the EM field due to electron-ion collisions can be calculated. For a BL equal to or less than $1 \mathrm{~cm}$, it would be about $1 \mathrm{~s}$ or less.

2. Many types of ions, atoms and molecules would exist in the plasma layer and these would vary depending on conditions. Although the electrons are extremely energetic, the ions would remain relatively cool due to heat conduction outward to the atmosphere. Hence, it is not thought that fusion would be possible.

3. Many EM modes are possible - electric and magnetic, multiple $n$, and multiple electric-field maxima between the core and electron layer (see Stratton, 1941; Budden, 1961a). Hence, nulls in the electric field are unlikely; however, electric field minima could exist.

4. Once the modes are established, the ball diameter would remain almost constant since size is almost independent of the total energy. In Eq. (13) the second term is due to atmospheric pressure and is negligible compared the first term for ES energy which is independent of $b$. Hence the ball radius $b$ is almost constant as the total energy decreases.

5. Mode interference might allow some of the electrons in the electron layer to pass through the EM barrier at electric-field minima. Their ES energy would be supplied continuously to the ball. Once through the EM barrier, these electrons would become relativistic as they spiral into the core. They would radiate light and ultra violet (cf. synchrotron radiation). Upon collision with the core, subatomic particles could be produced. Infra-red radiation would be negligible; hence, as has often been observed, no heat would be felt by a close observer.

6. The electron layer is attracted to the positive core. This holds the ball together and prevents it from diffusing away. In other models of BL, diffusion of the ball into its surroundings would occur rapidly.

Acknowledgements. Topical Editor F. D'Andrea thanks two anonymous referees for their help in evaluating this paper. 


\section{References}

Abrahamson, J.: Ball lightning from atmospheric discharges via metal nanosphere oxidation: from soils wood or metal, Phil. Trans. R. Soc. Lond. A, 360, 61-88, 2002.

Abrahamson, J. and Dinniss, J.: Ball lightning caused by oxidation of nanoparticle networks from natural strikes on soil, Nature, 403, 519-521, 2000.

Altschuler, M. D., House, L. L., and Hildner, E.: Is ball lightning a nuclear phenomenon?, Nature, 228, 545-546, 1970.

Ashby, D. E. T. F. and Whitehead, C.: Is ball lightning caused by antimatter meteorites?, Nature, 230, 180-182, 1971.

Banks, P. M. and Kockarts, G.: Aeronomy, Academic, Orlando, Fla., 1973.

Barry, J. D.: Ball Lightning and Bead Lightning, Prenum Press, New York, 1980.

Budden, K. G.: The Wave-Guide Mode Theory of Wave Propagation, Prentice-Hall Inc., Englewood Cliffs, N. J., 1961a.

Budden, K. G.: Radio Waves in the Ionosphere, Cambridge Univ. Press, New York, 1961b.

Charman, W. N.: Ball lightning, Phys. Rep., 54, 261-306, 1979.

Corliss, W. R.: Lightning, Auroras, Nocturnal Lights, and Related Luminous Phenomena, pp. 162-163, Published by the Sourcebook Project, P.O. Box 107, Glen Arm, Maryland, 1982.

Dawson G. A. and Jones, R. C.: Ball lightning as a radiation bubble, Pure Appl. Geophys., 75, 247-262, 1969.

Dikhtyar, V. and Jerby, E.: Fireball ejection from a molten hotspot to air by localized microwaves, Phys. Rev. Lett., 96, 045002-1045002-4, 2006.

Dmitriev, M. T.: Stability mechanism for ball lightning, Sov. Phys. Tech. Phys., 14, 284-289, 1969.

Dmitriev, M. T., Bakhtin, B. I., and Martynov, V. I.: Thermal effects of ball lightning, Sov. Phys. Tech. Phys., 26, 1518-1520, 1981.

Endean, V. G.: Ball lightning as electromagnetic energy, Nature, 263, 753-754, 1976.

Fejer, J. A.: Ionospheric modification and parametric instabilities, Rev. Geophys., 17, 135-153, 1979.

Golka, R.: Laboratory-produced ball lightning, J. Geophys. Res., D99, 10679-10681, 1994.

Goodlet, B. L.: Ball and bead lightning, J. Inst. Elec. Eng., 81, 1-56 (see pp. 32 and 55), 1937.
Hopple, W. A.: Theory of the electrode effect, J. Atmos. Terr. Phys., 29, 709-721, 1967.

Jones, A. T.: A laboratory illustration of ball lightning, Science, 31, 144, 1910.

Koslov, B. N.: Maximum energy liberation by ball lightning, Sov. Phys. Dokl., 23, 41-42, 1978.

Kuhn, E.: Ein Kugelblitz auf einr Moment-Aufnahme?, Naturwiss., 38, 518-519, 1951.

Meunier, M. S.: Substance singulière recueillie à la suite d'un météore rapporté à la foudre, Comptes Rendus, 103, 837-840, 1886.

Muldrew, D. B.: The physical nature of ball lightning, Geophys. Res. Lett., 17, 2277-2280, 1990.

Paiva, G. S., Paväo, A. C., de Vasconcelos, E. P., Mendes Jr., O., and da Silva Jr., E. F.: Production of ball-lightning-like luminous balls by electric discharge in silicon, Phys. Rev. Lett., 98, 048501-1-04508-4, 2007.

Potts, L. M.: Ball lightning, Science, 31, 144, 1910.

Schmidt, G.: Virial theorem for plasmas, Phys. Fluids, 3, 481-482, 1960.

Silberg, P. A.: Ball lightning and plasmoids, J. Geophys. Res., 67, 4941-4942, 1962.

Singer, S.: The Nature of Ball Lightning, Plenum Press, New York, 1971.

Smirnov, B. M.: Observational properties of ball lightning, Sov. Phys. Usp., 35, 650-670, 1992.

Spitzer Jr., L.: Physics of Fully Ionized Gases, Interscience Publishers, New York, p. 24-25, 1962.

Stephan, K. D. and Massey, N.: Burning molten metallic spheres: one class of ball lightning?, J. Atmos. Solar Terr. Phys., 70, 1589-1596, 2008.

Stratton, J. A.: Electromagnetic Theory, McGraw-Hill, London, 1941.

Turpain, M. A.: Curieux effets d'un coup de foudre sur une antenne réceptrice d'onde électriques, J. de Physique, 1, 372-382, 1911.

Webber, H.: A case of globular lightning, J. Roy. Astron. Soc. Can., 1, 44, 1907. 